\title{
DE NACIÓN A ESTAR SIN NACIONALIDAD: SUJETOS MIGRATORIOS EN LA BIOREGIÓN AMAZÓNICA
}

\section{FROM NATIONHOOD TO NON-NATIONHOOD: MIGRATORY SUJECTIVITIES IN THE AMAZON BIOREGION}

\author{
Jacob G Price \\ The State University of New Jersey - Rutgers
}

\section{RESUMEN}

Como la construcción de la identidad basada en la nacionalidad va perdiendo importancia, es indispensable analizar otras maneras de formular la identidad que toman su lugar. En el caso de la región Amazónica, el concepto de una bioregión, que se define no por alguna especie de patriotismo o fronteras políticas sino por un ecosistema particular y la interacción de individuales dentro del ecosistema, mejor explica cómo los residentes amazónicos forman una comunidad e identidad. La bioregión, porque se trata de fronteras geográficas o naturales, entiende mejor la cosmología amazónica, la cual se basa en el ecosistema en que residen los humanos y no humanos.

Palabras clave: Bioregión, indígena, Amazonia, identitdad

\begin{abstract}
As identity based nationality is losing its importance, it is increasingly important to analyze other ways in which identity formation takes its place. In the case of the Amazonian region, the concept of a bioregion, defined not by any patriotism or poltical boundaries, but by a particular ecosystem and the interaction of individuals in that ecosystem, better explains how those who reside in the Amazon form a community and identity. Bioregions, because they deal with geographic or natural boundaries, encompasses Amazonian cosmology, which is based on the ecosystem which humans and non-humans inhabit together.
\end{abstract}

Keywords: Bioregion, indigenous, Amazon, identity 
La declaración que Arjun Appadurai dio en 1993 todavía no se ha resuelto por completo en el nuevo milenio: "We need to think ourselves beyond the nation" (158). Veintidós años después de la primera publicación de Public Culture, el trabajo de repensar cómo se analiza el sujeto fuera de las fronteras impuestas por la nación marcha adelante poco a poco. Hasta Appadurai propuso "that we begin to think of the configuration of cultural form in today's world as fundamentally fractal" (46), aludiendo a la necesidad de reemplazar la nación por componentes culturales. La ecocrítica, que tiene sus raíces alrededor de las publicaciones de Apparudai, ha sugerido varias maneras de repensar la nación y la identidad humana no sólo por un componente cultural, sino en términos de la relación del ser humano con su ambiente. La noción de la bioregión sirve para entender la identidad amazónica y la relación entre la Amazonia y los que se identifican como parte de la Amazonia.

Tal como las fronteras políticas de Perú, Ecuador, Columbia, Venezuela, Bolivia, Surinam, Guyana, Guyana Francesa y Brasil reclaman parte de la Amazonia, ningún país es capaz de reclamar todas las epistemologías y ontologías amazónicas como la bioregión puede. La bioregión, un concepto que se ha dispersado en los estudios ecológicos y ecocríticos, es útil para comprender una identidad compuesta de epistemologías y ontologías indígenas que no cabe dentro del discurso nacional. Cheryll Glotfelty ofrece esta definición de la bioregión en su libro más reciente: "Bioregions are geographic areas having common characteristics of soil, watersheds, climate, and native plants and animals that exist within the whole planetary biosphere as unique and intrinsic contributive parts... Everyone lives in some bioregion or other" (62). Además, la bioregión se entiende por fronteras geográficas que naturalmente divide la tierra, por ejemplo montañas, bosques y ríos. Debido a que la característica totalizante de la nación poco a poco va despareciendo del las conversaciones académicas, es indispensable rearticular la identidad espacial con que las personas, y en particular personas o sujetos migratorios, se autodefinen y son definidos. 
Las fronteras políticas que impone la nación pierden su poder convincente, volviéndose arbitrarias al hablar sobre la migración. John Lie, comentando sobre la crítica de Gino Speranza, propone que: "As the twentieth-century American Gino Speranza (1925:163) declared: "We know that a republic is posible only to men of homogeneous race." However, no where has it reached this condition. Indeed, the ideal of one state, one people is unattainable" (145). Lie se refiere a las migraciones masivas entre naciones a partir de la colonización y arguye que estas migraciones masivas han existido y sido fundamentales en la construcción de la civilización y nación (146). La diáspora deshace la noción de la nación porque la llegada de un sujeto migratorio da a luz el carácter excluyente y arbitrario del proyecto nacional aunque la nación predica una narrativa totalizante que supuestamente incluye a todos.

Esta narrativa totalizante ha sido una fuerza indispensable en el contexto de América Latina. En el libro importante de Doris Sommers, Foundational Fictions, ella sugiere que el discurso nacionalista y el género novelista forman el cimiento sobre el que se construye la comunidad imaginaria de una nación (7). Sommers se concentra en el poder legislativo y militar de los criollos blancos masculinos, per es importante destacar que el gobierno que representa este poder es capitalista. Así que la narrativa creada y diseminada por las novelas nacionalistas en Latinoamérica llevan la carga de la epistemología y ontología basadas en el capitalismo. Sommers menciona las tensiones entre el estado, el mercado y la necesidad de crear una historia nacional pero también señala que estas tensiones están mitigadas por el amor romántico simbolizando el amor patriótico (51). Sin embargo, en el caso de la diáspora, no solo se trasladan personas, sino epistemologías, ontologías e ideologías que pueden derrumbar la nación construída. Así que en la deconstrucción del discurso nacionalista y novelista que presenta Sommers, Cuentos amazónicos, y en particular los cuentos "El regalo de la Yara" y "La ciudad encantada", de Juan Carlos Galeano, es una colección de cuentos folklóricos escrita desde una epistemología y ontología indígenas y nativas a la Amazonia, que desafía a la nación y a la novela nacionalista tal como los criollos americanos las idearon en el siglo XIX. Esta colección en particular y la bioregión amazónica que representa, están en conflicto total con las novelas nacionalistas y también las novelas de la tierra de los siglos XIX y XX que intentan dividir y luego unir la nación a través del binarismo civilización/barbarie al domar al "bárbaro".

Las fronteras geográficas de la bioregión amazónica y las fronteras políticas de las naciones desde que se escriben las novelas nacionalistas y las novelas de tierra se traslapan en la selva amazónica. Lo más curioso de la bioregión amazónica es que en la bioregión amazónica existen la 
epistemología, cosmología, y ontología indígenas que unen a los que viven en La Amazonia mejor que el discurso nacional. La razón principal por la que es más útil acudir a la bioregión para buscar una identidad o espacio a que y desde que se traslada la gente, es precisamente por las creencias compartidas en la bioregión amazónica que retan el nacionalismo. Es decir, porque un grupo o unidad social se define por su epistemología y ontología compartidas, el caso de la bioregión amazónica sirve mejor que el caso nacional para definir al grupo social. Los grupos indígenas de la Amazonia ejemplifican una identidad basada en la ontología compartida.

El cuento titulado "El regalo de la Yara" de Cuentos amazónicos, se gira en torno a la diferencia de ontología entre la ontología indígena amazónica y la ontología occidental y capitalista. El cuento relata cómo "un joven de Lima" llega a la Amazonia y tiene un encuentro con una Yara, que es un delfín de la mitología amazónica, que llega a su casa durante la noche y le regala un pez dorado (Galeano, 41). El joven, no entendiendo la significancia del pez dorado, no lo acepta y los indígenas le dicen después que debía de recogerlo porque habría traído suerte al joven.

El cuento comienza con una frase que indica la ontología occidental y capitalista del joven: "Un joven de Lima quería volverse rico en Amazonas" (41). Siendo el sujeto hombre y joven, ya hay claras semejanzas entre el hombre del cuento y los hombres de las novelas nacionalistas. Porque es de Lima, representa la ciudad y la modernidad. El único motivo del hombre joven es "volverse rico", lo cual no solo destaca su pensamiento capitalista sino también su capitalismo en conjunción con los recursos naturales de la naturaleza. Este discurso alude al análisis proporcionado por la ecocrítica poscolonial, que critica la visión capitalista del desarrollo de países "menos desarrollados" para perpetuar la teoría de dependencia de América Latina a costo del ambiente (Huggan y Tiffin, 30). El hombre joven de Lima, siendo un capitalista, busca su riqueza "en Amazonas" y no en otro lugar. Al ir a la selva para "volverse rico", el joven percibe la naturaleza como mercancía en lugar de un espacio donde los seres vivientes coexisten porque todos tienen espíritu humano. Esta perspectiva marca el conflicto de las epistemologías y ontologías occidentales e indígenas en la bioregión amazónica. Porque el "joven de Lima quería volverse rico en la Amazonia", se destaca de los demás residentes de la bioregión por su intención capitalista. Puesto que la ontología indígena cree en un balance en el ecosistema entre todas las entidades naturales por su agencia comunicativa compartida (Uzendoski, 23), la idea de "volverse rico" no cuenta con la ayuda de la naturaleza para hacer esto, sino que como lenguaje capitalista, presenta la violencia de extraer los recursos en lugar de cooperar y trabajar con la tierra.

Además, el joven peruano, "para lograr [volverse rico], se fue a sacar 
madera en el Manú, donde hizo campamento y contrató a gente de la zona" (Galeano, 41). La migración del joven peruano a la Amazonia es llamativa porque se basa en el cruzar fronteras políticas e ideológicas. La explicación de Simon Stander sobre la conexión entre el capitalismo y la nación es útil para comprender cómo el joven peruano se define tanto por la nación que cabe dentro de la ontología occidental como la ideología capitalista:

"These frontiers [between nation-states] are entirely man-made, or, more correctly, system-made. They have been created by a political and economic system that began in Western Europe and have been aided by forms of capitalism with its paradoxical tendency to transcend frontiers, creating supra-territorial space for its special manner of exploitation and creation of surpluses." (69)

La relación entre el pensamiento occidental, el capitalismo y las fronteras políticas que señala Stander es sumamente importante para entender cómo el joven peruano representa la idea de la nación al migrar a la bioregión amazónica. Esto se hace claro en la descripción que la narración da del hombre joven diciendo que "se fue a sacar madera en el Manú, donde hizo campamento y contrató a gente de la zona" (41). Acudiendo a la cita de Stander, el hombre joven, con sus motivos captialistas, crea un "supra-territorial space" para poder explotar y generar dinero. Este espacio, que intenta vincular la nación de Perú con la bioregión amazónica a través del capitalismo, tiene como su fin hacerle rico al joven peruano sin preocuparse por el medio ambiente, las personas y las creencias indígenas que ya coexisten en la Amazonia con la naturaleza y la cosmología indígena.

Aunque en el cuento no hay una crítica de la riqueza en sí ni a la manera en que se extraen los recursos naturales, el cuento demuestra cómo la epistemología indígena vale más para asimilarse y morar en la bioregión amazónica, lo cual sugiere que para ganarse la vida, es necesario conformarse a la ontología indígena. Durante la noche en que la Yara va a su casa, el hombre joven "tuvo tanto miedo (lo que habría de pesarle siempre) que se pegó a la cama agarrado a un crucifijo... Al fin pudo dormir apretando el diente de delfín que le había regalado uno de los indígenas" (41). El discurso religioso, que informa la mitología y la ontología, destaca cómo la ontología indígena no sólo prevalece en la bioregión sobre la ontología occidental o cristiana, sino que distingue a las personas de afuera con las que residen en la Amazonia. Esto es obvio en la yuxtaposición del crucifijo con el diente de delfín. Los dos tienen la misma función de traer paz a la 
persona, pero únicamente sirve el diente de delfín para el hombre joven porque el miedo que tuvo el joven era a la Yara, una cosa que cae dentro de la mitología indígena. El crucifijo que promete protección a los demonios en la mitología cristiana no sirve para ayudar al hombre joven con la Yara. La epistemología y ontología que trae el hombre joven a la Amazonia tienen un conflicto con la epistemología y ontología indígenas de la bioregión, impidiendo la asimilación total del sujeto migratorio en la Amazonia.

Aun después de exitosamente acudir a la mitología y ontología indígenas para superar el miedo que tuvo, esta acción presenta cómo el joven convive con la ontología indígena pero nunca confía completamente en la epistemología indígena hasta integrarse en la bioregión. Por esa falta de asimilación total a la bioregión amazónica, él, aunque reside en la bioregión, se distancia de ella con el rechazo de un regalo de la Yara. El joven "por una rendija de la puerta vio un pez hermoso revolcándose en la tierra del patio. Era un dorado. Quiso salir a cogerlo pero no lo hizo" (42). La Yara que dejó un pez dorado fuera de la casa del hombre funciona como la epistemología indígena que intenta llegar al hombre joven. La "rendija de la puerta" actúa como una barrera entre el hombre joven y el regalo de la Yara. Aunque él reside en la Amazonia en una casa, rechaza lo que la bioregión le ofrece al ver el pez dorado y no "cogerlo". Percibe la ontología indígena y la identidad bioregional amazónica, pero al final las rechaza, y por eso no se hace parte de la bioregión.

Esta distinción es fundamental para entender al hombre joven como un sujeto migratorio. Luego en el mismo día en que rechaza el regalo de la Yara, el hombre joven cuenta a sus trabajadores de lo sucedido y uno de ellos le responde que "pudo haber sido la Yara quien había venido a enamorarlo. La detuvo ese diente de delfín que lleva usted en el cuello. Pero téngalo por seguro, patrón, que le va a ser bien difícil enamorarse de otra mujer. No importa a qué ciudad o país usted vaya"" (42). La acción de enamorar indica la conexión íntima que el joven podía haber tenido con la cosmología indígena y que la Yara, siendo parte de la bioregión amazónica, intentaba hacerle parte de la bioregión amazónica también. Cuando el trabajador indígena dice que "le va a ser difícil enamorarse de otra mujer", volviendo al discurso de la novela nacionalista y el nacionalismo, la mujer a menudo no solo simboliza la tierra en sí, sino la nación que ha sido civilizada por el hombre también. Entonces, después de morar en la bioregión amazónica y conocer a la Yara, una mujer de la cosmología amazónica, el joven nunca la puede dominar como los protagonistas masculinos en las novelas nacionalistas. Es decir, aunque el joven peruano, que representa a la nación por su deseo de "volverse rico" a través del capitalismo, intenta explotar la madera de la selva amazónica, queda fracasado 
en su proyecto porque tenía que conformarse a la cosmología indígena para lograrlo cuando los protagonistas de las novelas nacionalistas no se conforman a nada, sino que hacen conformar a otros. Sin embargo, si un sujeto migratorio se incorpora en la cosmología indígena, la búsqueda de las riquezas se vuelve mucho menos importante. Cómo la incorporación en la cosmología indígena logra efectuar este cambio se tratará más en "La ciudad encantada".

De hecho, la figura de la Yara va en contra de las novelas nacionalistas porque intenta seducir al protagonista masculino para que él se integre a la cosmología indígena sin que el hombre joven pueda seducir a la Yara. Por eso, el hombre peruano ni tiene la oportunidad de dominar a la Yara por su falta de conocimiento de la cosmología indígena. Los papeles del hombre civilizador y la mujer bárbara de las novelas nacionalistas de una manera se ponen al revés: el hombre de la nación exitosamente es seducido por la mujer de la selva. La diferencia clave entre Cuentos amazónicos y las novelas nacionales es que la mujer Yara es narrada no cómo bárbara ni civilizadora sino cosmológicamente (ex) humana para mostrar una igualdad entre el hombre y su ambiente en la naturaleza. Entonces, "El regalo de la Yara" presenta un discurso ecológico basado en la ontología indígena y no un discurso nacionalista basado en el capitalismo.

Al final, es su rechazo que le aleja al joven de la bioregión amazónica aunque al aceptar el regalo de la Yara, el joven habría sido dominado por la ontología indígena. Además, por que "le va a ser difícil enamorarse de otra mujer", será difícil reintegrarse a una nación después de vivir una temporada como partícipe de una bioregión. Michael Uzendoski explica que "as the story points out, the encounter with the fish-woman changes the man forever, for his body and soul have been taken over by his desires flowing into the unseen world" (60). Porque una parte del joven peruano se siente atraída a la cosmología indígena, el retorno a su país de origen se dificulta. Este retorno difícil, sin embargo, no será de nacionalidades sino de epistemologías y ontologías occidentales y urbanas porque son ontologías que están en conflicto, no nacionalidades.

El trabajador indígena enfatiza la asociación entre la mujer y la nación además de la distinción de las ontologías al agregar que "No importa a qué ciudad o país usted vaya" (42). Aunque el uso del subjuntivo implica un futuro incierto para el joven, alude a que el joven no se quedará en la bioregión amazónica, y esto debido a su rechazo del regalo. La Yara es única, y es una identidad que no puede encontrar en ningún otro lugar. Entonces al rechazarla, rechaza también la identidad bioregional amazónica por una identidad de "ciudad o país". 
Cuando el joven pide una explicación del pez dorado, el trabajador indígena comenta que "Le iba a traer buena suerte" (42). Al decir que "le iba a traer suerte" (énfasis mío), el trabajador indígena se refiere al hecho de que era posible que el joven tuviera éxito en la selva amazónica pero que ahora ya no existe esta posibilidad. El trabajador enfatiza que el joven perdió una oportunidad para integrarse a la bioregión con la siguiente oración: "Usted debió salir a cogerlo, patrón" (42). Además que una repetición de la pérdida de oportunidad, el trabajador emplea lenguaje formal y capitalista para marcar una división ideológica. La repetición del título "patrón" y el pronombre formal "Usted" a lo largo del cuento llama atención a la distinción de clase socioeconómica entre el trabajador y el joven y distinción de ontología. Esta distinción viene del capitalismo, y no de la cosmología indígena. El uso del título "patrón" para cerrar el cuento lleva un tono irónico porque su posición en la oración viene después del consejo que el trabajador indígena le da al hombre joven, cuando la jerarquía capitalista sugerida por el título "patrón" pone al hombre joven por encima del indígena. Entonces, el capitalismo intenta imponerse en la ontología indígena con el título "patrón", pero el conocimiento de la cosmología indígena tiene la última palabra porque es el trabajador indígena con su conocimiento de la cosmología indígena que aconseja al joven. Como el joven no recibió el regalo de la Yara, no se hace parte de la comunidad amazónica sino que simplemente reside entre los de la comunidad amazónica.

Aunque el cuento se concentra en la inhabilidad del joven para integrarse en la comunidad amazónica y alude a su retorno, la noción de la bioregión marca bien cómo una gente no solo puede vivir fuera las fronteras nacionales y el poder del estado ejercido a través del capitalismo sino que desafía la necesidad de acudir a la nación para identificase. Es útil examinar cómo una persona definida por su nacionalidad entra en una región que se entiende mejor por su identidad bioregional. Al yuxtaponer un sujeto migratorio identificado por su nacionalidad con una bioregión, se destacan la arbitrariedad de la nación y sus fronteras y la utilidad de ver cómo una gente se entiende por fronteras naturales, inscritas en la geografía misma de un lugar.

"La ciudad encantada" relata cómo otro hombre peruano llega a la Amazonia pero en lugar de encontrarse con una Yara, encuentra a una mujer que le muestra una ciudad utópica. El cuento comienza de una manera parecida a "El regalo de la Yara" con otro hombre peruano, esta vez de lquitos, que quiere trabajar como maderero y ganar dinero.

Desde los dos primeros párrafos, hay indicaciones de que este joven de Perú no lleva la mentalidad capitalista a la Amazonia porque va allá "para 
apoyar a sus padres" (47). La diferencia entre el hombre de "El reglado de la Yara" y el joven de "La ciudad encantada" es marcada por el egoísmo - la falta de egoísmo y el avaricio o la falta de avaricio. El propósito de la migración del joven peruano de la segunda historia es sostener a su familia, lo cual indica que entiende y cree en la idea de comunidad. En la próxima oración, la narración comparte que "después de las labores, mientras los otros jugaban cartas para distraerse, él se alejaba por la orilla del río" (47) lo cual muestra cómo el joven no sólo apreciaba a la comunidad familiar sino que prefería pasar tiempo a solas en la naturaleza en lugar en la compañía de los trabajadores.

Porque "él se alejaba", el joven peruano crea una distancia entre él y los demás trabajadores, que forman una comunidad basada en la labor o el dinero, y también se acerca al río, que en la ontología indígena es una comunidad de seres humanos y ex-humanos. La disposición ideológica del hombre de entender el mundo y la necesidad de los humanos en términos de la comunidad no basada en el dinero antes de ir a la Amazonia le prepara para interactuar con la cosmología amazónica: "Así fue como se encontró con una muchacha que era toda una belleza" (47 énfasis mío). Su entendimiento de las comunidades que van más allá de la labor o el dinero, le pone en un lugar particular en que encuentra a una mujer de la cosmología amazónica. Es decir, la perspectiva diferente sobre las comunidades por sí misma no le presenta a la mujer, porque vemos que en "El regalo de la Yara", la perspectiva capitalista no le presenta a la Yara, sino la proximidad de la naturaleza. Lo que difiere a los dos hombres es cómo interactúan con la cosmología amazónica cuando está en su presencia. El muchacho de lquitos en el segundo cuento "después de verla varias veces e ilusionarse son ella, se enamoraron" (47). Resalta otro contraste importante de los dos cuentos porque el amor entre el muchacho de lquitos y la mujer es mutuo y exitoso. Más interesante es que al contrario de las novelas nacionales, el hombre no intenta "civilizar" a la mujer de la selva. Su amor no presenta un discurso patriarcal para construir la nación y domar a la barbarie. De hecho, después de que los dos hablan sobre las ciudades de las que son, es ella quien le invita a visitar a su familia y él luego acepta. Su encuentro con la mujer y la invitación de participar de una comunidad de la cosmología indígena señala que el muchacho peruano es capaz de entrar en la cosmología indígena.

Porque la ontología indígena en la bioregión amazónica permite que cualquier persona se identifique como parte de la bioregión amazónica, es importante ver cómo el muchacho peruano se integra a esta bioregión a través de la cosmología amazónica. Para persuadir al peruano a que vaya con ella a visitar su ciudad y a su familia, la mujer "se puso a describirle 
el lugar, diciendo que allá la gente no se mataba trabajando. Las casas tenían sus despensas llenas de comida y se servían con platos de oro decorados con piedras preciosas" (47). La retórica que la mujer emplea tiene mucho que ver con ideologías capitalistas que presentan el descanso y la riqueza como la recompensa del trabajo. Parece que ella utiliza esta retórica para presentar su comunidad utópica de modo que el muchacho peruano, siendo un hombre del mundo occidental y capitalista, puede comprenderla.

Sin embargo, lo que ella le invita en realidad es entrar en la ontología y cosmología indígenas de la Amazonia aunque la retórica parece capitalista. La ciudad que ella describe más bien cae en la cosmología indígena porque para llegar a la ciudad de donde es la mujer, los dos tienen que pasar por varias barreras protegidas por la vegetación y los animales, lo que señala lo que Jeremy Larochelle dice sobre la cosmología amazónica: "This worldview does not hold humans at the center but rather acknowledges the interconnectedness between human and non-human, between concrete reality and other spheres that exist simultaneously" (200). Cuando se enfrentan a la primera barrera, "Ella le dijo algo a la planta y la puerta se abrió" (Galeano, 47) y en el momento que llegan a la segunda barrera, "... un portón guardado por dos árboles renaco con boas enredadas en las ramas. [Ella] les silbó y los dejaron pasar" (47). Estas escenas breves en que la mujer interactúa con la vegetación y los animales rematan cómo la bioregión amazónica se comprende por la cosmología y ontología indígenas. Es un ecosistema en que todos los seres vivientes tienen alma y pueden comunicarse e interactuarse para el provecho de todos. La interconectividad entre la mujer y los animales y la vegetación ejemplifica lo que Eduardo Viveiros de Castro ha dicho sobre la ontología amazónica indígena, que es el espíritu humano entre todos los seres vivientes que permite una comunicación y entendimiento entre todos: "For Amazonian peoples, the original common condition of both humans and animals is not animality but, rather, humanity" (465). Las implicaciones de la ontología indígena son esenciales para entender que, por extensión, la preocupación del origen nacional del ser humano no tiene importancia en la Amazonia. La llegada de una persona desde fuera de la bioregión amazónica, como el joven peruano, no determina su estatus como sujeto migratorio puesto que ni el estado vegetal o animal no se distingue del ser humano, sino que son las fronteras políticas que definen si uno es un sujeto migratorio o no.

La primera implicación de la ontología indígena, entonces, es que todos los seres humanos, sean de donde sean, se consideran seres humanos al igual que "Animals [and plants] are ex-humans (rather than humans, ex-animals)" a la vista indígena (Viveiros, 465). Lo que sí distingue a una persona que viene desde fuera de la bioregión amazónica para habitarla 
es cómo la ontología occidental trata a los animales y plantas como entidades distintas de los seres humanos. La ontología occidental separa los seres humanos de los animales y plantas por el discurso científico basado en la teoría darwiniana de evolución. Vivieros se refiere a esto como "our own "scientific" mythology" (465) con el propósito de igualar la ontología indígena con la ontología occidental mediante la noción de la mitología. El miedo que sentía el hombre peruano de "El regalo de la Yara" durante la noche se debe a una música que escuchaba desde el río y él intenta usar la "mitología científica" para entenderla. El joven intenta determinar de donde viene la música cuando "fue a indagar y era el ruido del agua rozando el barranco" (41). Al "indagar" sobre la fuente de la música, el hombre peruano está acudiendo a la ciencia, determinada por los cinco sentidos, para averiguar qué está pasando y concluye que la música no está, sino que sólo es el agua que hace ruido. Acude al oído para llegar a esta conclusión, y luego a la vista cuando "se volvió a dormir otra vez pero la música continuó. Salió y no vio a nadie" (41). Como el hombre peruano acude primeramente a la ciencia occidental para explicar un fenómeno de la cosmología indígena amazónica, no es capaz de comprender lo que está pasando fuera de su casa. El sujeto migratorio, si viene desde la nación viene de la ontología occidental también, no percibirá ni interactuará con la selva amazónica de la misma manera que los residentes de la bioregión. Es en esta diferencia que se crea el sujeto migratorio en la bioregión amazónica y no la nacionalidad.

Otra implicación de la ontología amazónica aparte de la humanidad universal en todo ser viviente es la noción de agencia que todo ser viviente puede ejercer. En armonía con el concepto de la bioregión que enfatiza la interconectividad de todo ser viviente en un ecosistema particular, la ontología amazónica reconoce que la humanidad que posee todo ser viviente abarca también la agencia de todo ser viviente. Alberti Benjamin y Yvonne Marshall explican que en la ontología amazónica: "what is significant is that people act towards things as if they had agency" (346). Según esta perspectiva, cuando una persona viene a la bioregión amazónica, se destaca de los demás humanos, sean seres humanos o ex-humanos, por la manera en que tratan al ambiente. Benjamin y Marshall contextualizan su estudio en el animismo, lo cual es significativo porque, y lo afirma Vivieros, es el espíritu humano que une a los ex-humanos con los humanos. Esto se ve claramente cuando la mujer hablar con la vegetación y las boas para entrar en su ciudad.

En cuanto llegan la mujer y el muchacho a la ciudad de donde es ella, la narración relata que "[al muchacho] lo asombró la belleza de las avenidas y de muchas fuentes. Allí se bañaban los habitantes para curarse de las enfermedades y alargar sus vidas" (48). La comunidad que ve convive 
felizmente y en abundancia. Tal como la mujer le había dicho al muchacho que sobra la comida, también sobra el agua y todos tienen buena salud. Esta abundancia es importante porque demuestra su comunidad estrecha y con muchos recursos sin el capitalismo para generar su abundancia. Los fuentes comunitarios que poseen alguna cualidad sanadora no requieren una entrada o dinero para usarlos. Todos gozan de ellos libremente. Entonces, si la gente "no se mataba trabajando" (47) se pregunta cómo todos pueden convivir felizmente sin el trabajo duro que el capitalismo enfatiza. La respuesta se encuentra en la noción de la cosmología indígena, en que existe una interconectividad entre todos los humanos, sean ex-humanos o no. Esta interconectividad forma un parte importante de la bioregión amazónica porque en esta bioregión el ecosistema proporciona las fronteras, y son estas fronteras con la ontología y cosmología indígenas que unen a los que residen en la Amazonia.

Al final del cuento, el muchacho deja atrás a su país natal, trabajo como maderero y a su propia familia, porque desea vivir en esta harmonía con la naturaleza, lo que señala cómo acepta la ontología indígena y el rechazo de la ontología occidental. La evidencia de esto se encuentra cuando el hombre peruano vuelve de la ciudad utópica a sus amigos para que sus amigos puedan

"enviarles a [sus] padres esta carta y esta bolsa de cuero.

...uno de sus compañeros, muy curioso, abrió la bolsa y halló que ésta contenía pepitas de oro.

Se quedaron asombrados.

Entonces los amigos, y otros que no lo conocían, decidieron salir del campamento para seguirlo y tener la misma suerte.

Pero no lo pudieron alcanzar." (48)

Porque el cuento termina con que el muchacho envía pepitas de oro a su familia y desparece en la selva, es evidente que para él, la riqueza, en el sentido capitalista, no ya le importaba. Entiende que sus padres todavía necesitan dinero para sobrevivir, entonces antes de irse para siempre, quiere darles lo que puede. Sin embargo, es importante notar que el muchacho de lquitos simbólicamente deja no solo el mundo capitalista atrás sino que también una vida y ontología que prestigian la búsqueda de una fortuna. Las pepitas de oro, que son la manifestación tangible de la riqueza, no le importan a él, pero a sus amigos y hasta "otros que no lo conocían" eran importantes.

Es llamativo que los amigos y los desconocidos busquen más oro porque 
muestra cómo el capitalismo se enfoca más en la riqueza que las personas mismas. Ni nos dice la narración si los amigos le trajeron las pepitas de oro a la familia del muchacho peruano, sino que se concentra en la reacción de las personas que no participan de la ontología y cosmología indígenas. Más importante que la reacción de los amigos y los desconocidos es la última oración "Pero no lo pudieron alcanzar" (48). El final definitivo del cuento concuerda con el final de la búsqueda de riqueza y también el límite de entendimiento de los que no subscriben a la ontología y cosmología indígenas. Para ellos, el muchacho peruano ha desaparecido con el conocimiento de dónde sacó las pepitas de oro en la selva. El final del cuento siguiere, entonces, una especie de frontera de conocimiento que no los amigos y desconocidos pueden cruzar o "alcanzar" porque su conocimiento está limitado a la ontología occidental y al avaricio capitalista. Ellos comentan que porque el muchacho se ausentó por mucho tiempo es "seguro que [esa mujer] es un fantasma o un alma del demonio" (48). Tal como en "El regalo de la Yara", hay un discurso religioso occidental al identificar a la mujer como un fantasma o un demonio. No hay evidencia de que ella sea una figura sobrenatural o mítica como la Yara porque se refiere a ella únicamente como una mujer. Ella simplemente es una mujer que participa de la bioregión amazónica, no de la nación ni ninguna nacionalidad, y entiende su alrededor según la ontología y la cosmología indígenas.

Es indispensable notar que la ontología y cosmología indígenas no les impiden cruzar a los amigos esta frontera porque tal como Uzendoski lo cita en su libro, Fernando Santos-Granero comparte que "boundaries are meant to be crossed" in the Amazonian world" (Uzendoski, 61). Esto es evidente con el muchacho peruano porque él, a la invitación de la mujer de la selva, ha podido cruzar las barreras protegidas por la vegetación y los animales y entrar en la ciudad encantada. Los amigos todavía viven en el mismo ecosistema que la mujer y el muchacho peruano, y así residen en la bioregión tal como el hombre joven de "El regalo de la Yara" reside en la bioregión, pero claramente no se integran a la bioregión como el muchacho de lquitos. La bioregión amazónica, en que se encuentra la ciudad encantada, entonces, se ha abierto al mundo occidental porque el muchacho pervano ha podido explorar las fronteras ontológicas y luego eligió integrarse a ella. Su cambio se basa en la ontología y no en las fronteras políticas.

En la migración, lo que se chocan en los conflictos entre el sujeto migratorio y el nativo del país o región de acogida son las epistemologías u ontologías diferentes, no las nacionalidades ni las fronteras políticas. Las nacionalidades se construyen a base de una epistemología u ontología particular que intenta imponerse al otro. Neil L. Whitehead explica que: 
"The idea of development in Western discourse alludes to form of both material and spiritual redemption and advancement and should be understood as part of the colonial and national conquest and incorporation of indigenous communities under the power of the state and government. Amazonia is thus seen as the end point of exploration, the counterpoint of modernity, and so the necessary context for development. Accordingly, language of the conquest and occupation still suffuses the national imagining of this region..." (107)

La violencia ontológica impuesta sobre el otro se ve claramente en las novelas nacionales que tenían el propósito de crear una comunidad imaginada llamada: la nación. Tal como Whitehead enseña y que Huggan y Tiffan describen, el discurso del desarrollo y la modernidad, que es central en las novelas nacionales son armas para crear una sociedad homogénea. Lo que estas novelas nacionales ignoran es la posibilidad de otro discurso que pueda crear otras identidades para unir a la gente. Frank Hutchins dice que "There are histories outside the history of capitalist forms that continue to influence subjectivities and frame responses to the demands of the state and the blandishments of the market" (6). Cuentos amazónicos es un libro que ejemplifica cómo la nación no ha podido ni nunca podrá crear la nación homogénea precisamente porque desafía la lectura nacional de las novelas nacionales y demuestra cómo sujetos migratorios desde la nación son influenciados por la ontología y cosmología amazónicas. "El regalo de la Yara" enseña cómo la nación fracasa en su tentativa de lograr explotar por completo a la Amazonía. "La ciudad encantada" demuestra que la nación no proporciona una identidad adecuada para todos sus sujetos individuales y que la bioregión amazónica es capaz de proveer una identidad más unificadora que incluye a los sujetos cosmológicos amazónicos. Cuentos amazónicos resalta la falsedad de la nación unida y enfatiza que la nacionalidad no sirve para mapear toda trayectoria diaspórica o migratoria. Estas trayectorias entre epistemologías y ontologías no se limitan a las fronteras e incluso, pueden ocurrir dentro de la nación misma. Sin embargo, desde la bioregión amazónica es posible entender los discursos nacionales de los países de la rodean. Los conflictos que surgen de la asimilación a una epistemología $u$ ontología nuevas se entienden mejor por un choque entre bioregiones que naciones. 


\section{Bibliografía}

ALBERTI, Benjamin and Yvonne MARSHALL. "Animating Archeology: Local Theories and Conceptually Open-Minded Methodologies." Cambridge Archeological Journal. 19.3. Cambridge. 2009. Impreso.

APPADURAl, Arjun. Public Culture. Chicago: U of Chicago. 1993. Impreso.

Modernity at Large: Cultural Dimension of globalization. Minneapolis: University of Minnesota P. 1996. Impreso.

GALEANO, Juan Carlos. Cuentos Amazónicos. Iquitos: Tierra Nueva. 2014. Impreso.

GLOTFELTY, Cheryll and Eve QUESNEL. eds. The Biosphere and the Bioregion. New York: $\quad$ Routledge. 2015. Impreso.

HUGGAN, Graham and Helen TIFFIN. Postcolonial Ecocriticism: Literature, Animals, Environment. London: Routledge. 2015. Print.

HUTCHINS, Frank. "Indigenous Capitalisms: Ecotourism, Cultural Reproduction, and the Logic of Capital in Ecuador's Upper Amazon" Editing Eden: A Reconsideration of Identity, Place, and Politics in Amazonia. Frank Hutchins y Patrick C. Wilson eds. Lincoln: U of Nebraska P. 2010. Impreso. LAROCHELLE, Jeremy. "Writing Under the Shadow of the Chullachaqui: Amazonian Thought and Ecologial Discourse in Recent Amazonian Poetry". Review: Literature and Arts of the Americas. 45.2. 2012. Impreso.

LIE, John. Modern Peoplehood. Cambridge: Harvard U P. 2004. Impreso. SANTOS-GRANERO, Fernando. Boundaries are meant to be crossed: The magic and politics of the long-lasting Amazon/Andes divide. Identities: Global Studies in Culture and Power 9.4: 545-69. 2002. Impreso.

SOMMER, Doris. Foundational Fictions: The National Romances of Latin America. Berkeley: U of California P. 1991. Impreso.

STANDER, Simon. Why War: Capitalism and the Nation-State. New York: Bloomsbury Publishing Inc. 2014. Impreso.

UZENDOSKI, Michael. The Ecology of the Spoken Word: Amazonian storytelling and shamanism among the Napo Runa. Urbana: $U$ of Illinois P. 2012. Impreso.

"Fractal Subjectivities: An Amazonian-Inspired Critique of Globalization Theory" Editing Eden: A Reconsideration of Identity, Place, and Politics in Amazonia. Frank Hutchins y Patrick C. Wilson eds. Lincoln: U of Nebraska P. 2010. Impreso.

VIVEIROS DE CASTRO, Eduardo. "Exchanging Perspectives: The Transformation of Objects into Subjects in Amerindian Ontologies." Common Knowledge. 10.3. Durham: Duke U P. 2004. Impreso. 
WHITEHEAD, Neil L. "Cannibal Tourists and Savvy Savages: Understanding Amazonian Modernities" Editing Eden: A Reconsideration of Identity, Place, and Politics in Amazonia. Frank Hutchins y Patrick C. Wilson eds. Lincoln: U of Nebraska P. 2010. Impreso. 